\title{
Changes in frequency of premature complexes and heart rate variability related to shift work
}

\author{
L G P M van Amelsvoort, E G Schouten, A C Maan, C A Swenne, F J Kok
}

Division of Human

Nutrition and

Epidemiology,

Wageningen

University, The

Netherlands

L G P M van Amelsvoort

E G Schouten

F J Kok

Department of Epidemiology,

Maastricht University,

PO Box 616, 6200 MD,

The Netherlands

L G P M van Amelsvoort

Leiden Foundation for ECG analysis SEAL,

Leiden University

Medical Centre, The

Netherlands

A C Maan

\section{Department of \\ Cardiology}

C A Swenne

Correspondence to:

Dr L G P M van Amelsvoort

Lgpm.vanamelsvoort@

epid.unimaas.nl

Accepted 7 June 2001

\begin{abstract}
Objectives-To investigate whether an increased risk of cardiovascular disease might be caused by increased arrhythmogeneity and by unfavourable changes in autonomic cardiac control the changes in the occurrence of premature complexes (PVCs) and in heart rate variability (HRV) were studied in subjects who started to work in shifts.

Methods-1 Year changes in frequency of PVCs and HRV were measured in 49 shift workers and 22 control subjects working in daytime. All respondents were starting in a new job in integrated circuit or waste incinerator plants.

Results-The incidence of PVC increased significantly in shift workers over the 1 year follow up, compared with daytime workers. The frequency of ventricular extrasystoles increased in $48.9 \%$ of the shift workers, and in $27.3 \%$ of the daytime workers. The Spearman correlation coefficient between the number of nights worked and the change in PVCs was $0.33(p=0.004)$. A small non-significant unfavourable change in HRV was found in both the shift and daytime workers. Conclusions-A change in arrhythmogeneity, but not in cardiac autonomic control, might explain the increased risk of cardiovascular disease in shift workers.

(Occup Environ Med 2001;58:678-681)
\end{abstract}

Keywords: arrhythmia; heart rate; shift work

Most of the studies on cardiovascular disease in shift workers are supportive of the hypothesis that they are at increased risk. In their review, Bøggild and Knutsson estimated the excess risk at $40 \% .^{1}$ Several explanations have been put forward, including disturbance of circadian rhythm, changes in behaviour, and disturbed sociotemporal activities. ${ }^{2}$ So far, about 40 studies have been reported that investigated the lifestyle and biological risk factors in shift workers. ${ }^{1}$ Although some of these studies reported a significant relation between risk of cardiovascular disease and shift work, it seems unlikely that these risk factors can fully explain the increased risk of disease in shift workers.

Research into other factors that may explain the increased risk of cardiovascular disease is scarce. Two of these factors are increased myocardial arrhythmogeneity and disturbed cardiac autonomic control. The frequency of ventricular premature complexes is an indicator of arrhythmogeneity. In men without coronary heart disease who had frequent or complex

\section{Main messages}

- The frequency of premature ventricular complexes (PVC) was found to be increased after 1 year of working in shifts, compared with working in daytime only.

- The increase in frequency of PVCs is related to the number of days worked on night shift (Spearman correlation 0.33, $\mathrm{p}=0.004$ ).

- No significant 1 year change in heart rate variability was found in shift workers.

Policy implications

- Increased arrhythmogeneity might explain, at least partly, the increased risk of cardiovascular disease in shift workers.

arrhythmias the relative risk of myocardial infarction or death from cardiovascular disease was estimated to be 2.30 (95\% confidence interval (95\% CI) 1.33 to 3.38). ${ }^{3}$ Kannel et al reported an increased risk of sudden death in both men and woman with ventricular premature beats. ${ }^{4}$ Heart rate variability is a noninvasive marker of cardiac autonomic control. In follow up studies, a high incidence of PVC (premature ventricular complexes) and unfavourable heart rate variability (HRV) have been related to increased cardiovascular morbidity and mortality. ${ }^{45}$ Tsuji et al reported a hazard ratio of 1.47 for new cardiac events (95\% CI 1.16 to 1.86 ) for a $1 \mathrm{SD}$ decrement in all RR intervals within a certain period $(\mathrm{SDNNi}) .^{5}$ The aim of the current study is to investigate whether working in shifts induces changes in heart rate variability and frequency of atrial and ventricular premature complexes. This might provide possible explanatory factors relating risk of cardiovascular disease and shift work. One year changes in these factors were compared between shift workers and daytime workers, all of whom had recently started their jobs.

\section{Methods}

POPULATION

The study population was a subset of participants from a cohort study of 396 shift workers and daytime working controls. The main objective of the cohort study was to assess for 1 year the influence of working in shifts on factors affecting the risk of cardiovascular disease. Due to practical restrictions only a subgroup of the total cohort received a Holter recording. These were a random selection of workers who started new jobs in either the 
Table 1 Baseline population characteristics and measurements

\begin{tabular}{|c|c|c|c|}
\hline & Day workers & Shift workers & p Value \\
\hline \multicolumn{4}{|l|}{ Population characteristics: } \\
\hline $\mathrm{n}$ & 32 & 75 & \\
\hline Age $(y$, mean $(S D))$ & $29.3(5.3)$ & $30.4(6.7)$ & 0.4 \\
\hline Sex ( $\%$ female) & 9 & 11 & $0.8^{\star}$ \\
\hline \multicolumn{4}{|l|}{ Smoking habits (\%): } \\
\hline Never smoker & 48 & 41 & \multirow[t]{3}{*}{$0.4^{\star}$} \\
\hline Current smoker & 26 & 40 & \\
\hline Ex-smoker & 26 & 19 & \\
\hline \multicolumn{4}{|l|}{ Education (\%): } \\
\hline Low & 3 & 38 & \multirow[t]{3}{*}{$0.001^{\star}$} \\
\hline Intermediate & 35 & 44 & \\
\hline High & 61 & 18 & \\
\hline \multicolumn{4}{|c|}{ Baseline frequency of premature complexes: } \\
\hline PAC (beats/h, median) & 0.54 & 0.47 & NA \\
\hline PAC (respondents with $>1 / \mathrm{h}, \%$ ) & 30.9 & 38.7 & $0.4 \dagger$ \\
\hline PVC (beats $/ \mathrm{h}$, median) & 0.043 & 0.042 & NA \\
\hline PVC (respondents with $>0.1 / \mathrm{h}, \%$ ) & 25.8 & 23.4 & $0.8 \dagger$ \\
\hline \multicolumn{4}{|l|}{ Baseline heart rate variability: } \\
\hline Heart rate (beats/min) & $71.6(8.4)$ & $75.3(8.4)$ & 0.04 \\
\hline SDNNi (ms) & $89.1(28.7)$ & $74.6(20.7)$ & 0.01 \\
\hline Loglow (log ms) & $-2.67(0.18)$ & $-2.76(0.21)$ & 0.03 \\
\hline Loghigh (log ms) & $-2.98(0.35)$ & $-3.15(0.30)$ & 0.03 \\
\hline$\%$ lf & $65.1(10.8)$ & $68.4(8.1)$ & 0.2 \\
\hline
\end{tabular}

${ }^{*} \mathrm{p}$ value by $\chi^{2}$ test.

tp value by Wilcoxon two sample test.

$\mathrm{PAC}=$ premature atrial complexes; $\mathrm{PVC}=$ premature ventricular complexes; $\mathrm{SDNNi}=$ mean of all 5 minute SDs of NN intervals; Loglow=power in the low frequency range $(0.05-015 \mathrm{~Hz})$ in logarithmic units; Loghigh=power in the high frequency range $(0.15-0.40 \mathrm{~Hz})$ in logarithmic units; \% lf $=$ low frequency power $(0.05-0.15 \mathrm{~Hz})$ in normalised units.

integrated circuit manufacturing industry or in waste incinerator plants in 1996 or 1997 . The ethics committee of Wageningen University approved the study; all respondents gave their written informed consent. Inclusion criteria were: starting in a new job; working for at least 32 hours a week; expecting to work for at least 1 year in the same job; no use of medication or previous admission to hospital for cardiovascular disease; no insurmountable objections against shift work (see measurements); and age between 18 and 55 years.

The 125 study participants (42 daytime workers and 83 shift workers) were asked to undergo two 24 hour Holter recordings, 1 year apart. Shift workers had their Holter recording during a day working in the morning shift and daytime workers during a usual working day. Thirteen respondents refused (eight daytime, five shift workers); one was excluded because of a second degree atrioventricular block; four had to be discarded because of technical problems such as excessive noise. This left 107 participants (32 daytime workers and 75 shift workers) who had a Holter recording at the start.

Seventeen participants refused to undergo the second Holter recording because "it was to cumbersome" (13) or because of skin irritation due to the electrodes (four). Fourteen were excluded because they had left their job. Five second Holter recordings could not be used because of technical problems. In total 71 complete pairs were used in the analysis (22 daytime workers and 49 shift workers).

DATA COLLECTION

Initial data collection was performed between 1 week and 2 months after the start of a new job, follow up data were collected 1 year later.

24 Hour Holter recordings

All subjects had a 24 hour Holter recording starting at the beginning of a morning shift (for the shift workers) or day shift (for the daytime workers). They were instructed to report start and end time of sleep, work, meals, leisure time, physical activities, and other possibly relevant events or activities in a diary. These diaries were coded afterwards according to a list of standard activities. Sleep was defined as 1 hour after going to bed until 1 hour before getting up, as recorded in the diary.

\section{Personal and work characteristics}

All subjects completed a questionnaire that considered personal and work characteristics. A detailed description of the measurements and characteristics used can be found elsewhere. ${ }^{6}$ Among others, the following characteristics were coded: education (coded in three levels); physical activity during work, sport, and leisure; smoking habits; current job; coffee consumption; shift work schedule;and job strain (job content questionnaire). In this study we defined shift work as working in an alternating work schedule including nights. Workers worked according to the medium speed backwards rotating shift and fast forward rotating shift, as defined elsewhere. ${ }^{7}$ For the stratified analysis the continuous variables were dichotomised, with the dividing point at the median.

\section{Assessment of heart rate variability}

The 24 hour Holter recordings were analysed with a Marquette Series 8000 Holter analyser by an experienced Holter analyst. The ventricular and atrial premature complexes were coded. A detailed description of the HRV analysis has been given elsewhere. ${ }^{67}$

\section{DATA ANALYSIS}

Because of the skewed distribution within and between subjects of the high and low frequency spectral HRV components, log transformation of these variables was performed. Standard methods were used for descriptive analysis. The distribution of the frequency of premature complexes and its 1 year change, did not approach a normal distribution, even after transformation. Therefore these differences were tested with the non-parametric Wilcoxon rank sum test. To evaluate the correlation with other continuous variables the Spearman rank correlation statistic was calculated. Potential confounding for these variables was assessed by stratified analysis. Regression analysis was used to assess the effect of other potential confounders on the transformed HRV variables. Seven respondents reported poor sleep quality during their first Holter measurement due to wearing the recorder for the first time. This had a significant influence on the HRV variables (for SDNNi: $p=0.004)$. Therefore differences in the 1 year change in HRV variables were adjusted for reported poor sleep quality by including a dummy variable for recordings where the people reported poor sleep quality in the regression analysis. All statistical analysis was done with the SAS program. 
Table 21 Year change of heart rate variability and frequency of premature complexes between baseline and the 1 year measurement

\begin{tabular}{|c|c|c|c|}
\hline & Day workers & Shift workers & $\begin{array}{l}p \text { Value of } \\
\text { difference between } \\
\text { day and shift } \\
\text { worker }\end{array}$ \\
\hline $\mathrm{n}$ & 22 & 49 & \\
\hline $\begin{array}{l}\text { Mean number of night shifts worked } \\
\text { between the baseline and repeated } \\
\text { measurement }\end{array}$ & 0 & 63 & \\
\hline \multicolumn{4}{|l|}{ Frequency of premature complexes (\%): } \\
\hline Decrease of PAC & 54.6 & 43.7 & $0.6+$ \\
\hline No change in PAC & 4.6 & 0.0 & \\
\hline Increase in PAC & 40.9 & 56.3 & \\
\hline Decrease of PVC & 45.4 & 20.4 & $0.03+$ \\
\hline No change in PVC & 27.3 & 30.6 & \\
\hline Increase in PVC & 27.3 & 48.9 & \\
\hline \multicolumn{4}{|l|}{ Heart rate variability: } \\
\hline Heart rate $(\mathrm{bpm})$ & 1.83 & $-2.08^{\star}$ & 0.08 \\
\hline SDNNi (ms) & $-7.0^{\star}$ & -2.0 & 0.2 \\
\hline Loglow $\left(10^{-3} \log \mathrm{ms}\right)$ & -36.9 & -29.9 & 0.84 \\
\hline Loghigh $\left(10^{-3} \log \mathrm{ms}\right)$ & $-83.9^{\star}$ & -16.2 & 0.06 \\
\hline$\%$ lf & 1.99 & -0.72 & 0.16 \\
\hline
\end{tabular}

$\mathrm{PAC}=$ premature atrial complexes $\mathrm{PVC}=$ premature ventricular complexes; $\mathrm{SDNNi}=$ mean of all 5 minute standard deviations of $\mathrm{NN}$ intervals; Loglow=power in the low frequency range $(0.05-015$ $\mathrm{Hz})$ in logarithmic units; Loghigh=power in the high frequency range $(0.15-0.40 \mathrm{~Hz}$.) in logarithmic units; \% lf $=$ low frequency power $(0.05-0.15 \mathrm{~Hz})$ in normalised units.

${ }^{\star} \mathrm{p}<0.05 v$ baseline.

tp Value according to Wilcoxon two sample test.

\section{Results}

In table 1 the population characteristics and baseline HRV and frequency of premature complexes are given. The only significant differences were higher educational level and heart rate, and a lower SDNNi and low and high frequency power in the shift workers compared with the daytime workers.

\section{PREMATURE COMPLEXES}

The 1 year changes in frequency of premature complexes and HRV are given in table 2 . The change in frequency of atrial premature complexes was not significantly different between the shift and daytime workers. The change in frequency of premature ventricular complexes was significantly different between the shift and daytime workers. The increase in premature ventricular complexes in shift workers was associated with the number of nights worked during the follow up period (Spearman correlation coefficient $0.33(\mathrm{p}=0.004))$. Stratified analysis according by smoking, young or old age, 1 year weight gain or loss, increase or decrease in alcohol consumption, high or low strain jobs, or good or poor sleep quality did not show substantial differences in the subgroups.

\section{HEART RATE VARIABILITY}

For the measures of $\mathrm{HRV}$ (SDNNi and frequency power levels) in daytime workers a significant decrease was found. In shift workers a significant decrease of 24 hour mean heart rate was found. None of these changes were significantly different between shift and daytime workers. Adjustment for changes in body mass index, coffee consumption, or job strain did not change the conclusions.

\section{Discussion}

In summary, we found that the 1 year change in frequency of ventricular premature complexes was greater in the shift workers than in the daytime workers. The changes in measures of variability in heart rate were not significantly different.

Given the cohort design, where each respondent serves as its own control, most potential sources of bias related to selection of the study population, are considered to be unimportant. However, selective drop out after the first measurement might have been a potential source of bias if this drop out was correlated with the 1 year changes. This form of selection bias could not be evaluated. However, considering the relatively few drop outs, together with the similar baseline values in the drop outs and the follow up population, selection bias is considered to be low. The choice of a control group remains problematic when investigating the effects of shift work. Although the cohort design is less sensitive to the choice of the control group, differences in the potential effects of working in shifts between shift workers and daytime workers might have influenced the results. Also differences in working conditions between shift workers and daytime workers might have occurred. However, a stratified analysis between high and low strain jobs, the most likely work related confounder, did not show different results between the groups. Furthermore the high correlation with the number of nights worked during follow up indicates that the increase in frequency of ventricular premature beats in the shift workers is due to working at night.

Although most of the change in variability of heart rate seems to be caused by regression to the mean, the baseline differences remain puzzling. It might be that this baseline difference reflects a selection effect. Because the first measurement was conducted after the start of a new job these baseline differences might also be related to adaptation to working in shifts. This effect might fade in time. Within the current data set it is not possible to decide which factor is responsible for this difference.

One other study reported on the relation between ventricular premature complexes and shift work. In this study by Härenstam et $a l^{\beta}$ working at night was related to an increased frequency of ventricular premature complexes, compared with working in the day. Both the study of Härenstam et al and our study support the involvement of increased susceptibility of the myocardium to arrhythmia in the risk of cardiovascular disease profile of shift workers. An increased frequency of ventricular premature complexes has been associated with an increased cardiovascular risk of disease. Bikkina $e t a l^{\beta}$ reported an adjusted relative risk of myocardial infarction and coronary heart disease of 2.12 (95\% CI 1.33 to 3.38 ) in men without cardiovascular disease with complex or frequent ventricular arrhythmia (more than 30 PVCs $/ h$ ).

Coffee consumption, body mass index, and smoking have been associated with the occurrence of ventricular premature complexes. However, when analysing these potential confounding factors in our study, they were not able to explain the reported increased frequency of premature ventricular complexes in 
shift workers. Speculating about a possible explanation for the increase, it might be that working at night acts as a chronic stressor. Theorell et al showed that a shift from day work to night work can induce acute sympathoadrenal arousal. Similarly, in a cross sectional analysis in shift workers we found evidence for a shift of the sympathovagal cardiac regulation towards sympathetic dominance during a night shift compared with a morning shift. ${ }^{7}$ In animal experiments it has been shown that exposure to social or psychological stress is related to an increased frequency of premature complexes. ${ }^{10}$ Also, in humans stress and fatigue have been related to an increased frequency in ventricular premature complexes. ${ }^{11}$

\section{Conclusions}

An increase in frequency of ventricular premature complexes during the first year of shift work, as found in this study, might explain the increased risk of cardiovascular disease in shift workers. A change in heart rate variability during days worked in the morning shift is probably not a factor in the mechanism leading to an increased risk of cardiovascular disease in shift workers. Further study is needed to confirm the results and to elucidate the factors involved in the increased frequency of ventricular premature complexes.

In our study only one of the shift workers reached $30 \mathrm{PVCs} /$ hour (the cut off level used in the study of Bikkinia et al). Nevertheless, we conclude that the increase in frequency of premature ventricular beats can be regarded as an indicator of unfavourable changes in the myocardial system, and should be regarded as a potentially important factor in the relation between shift work and the increased cardiovascular risk of disease.

This study was supported by grant 94.101 from the Netherlands Heart Foundation.

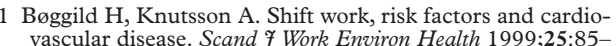
vascul

2 Knutsson A. Shift work and coronary disease. Scand F Social Med Suppl 1989;1-36.

3 Bikkina M, Larson MG, Levy D. Prognostic implications of asymptomatic ventricular arrhythmias: the Framingham heart study. Ann Intern Med 1992;117:990-6

4 Kannel WB, McGee DL, Schatzkin A. An epidemiological perspective of sudden death: 26 year follow up in the Framingham study. Drugs 1984;28 Suppl 1:1-16.

5 Tsuji H, Larson MG, Venditti FJJ, et al. Impact of reduced heart rate variability on risk for cardiac events. The Framingham heart study. Circulation 1996;94:2850-5.

6 Amelsvoort LGPM van, Schouten EG, Maan AC, et al. Occupational determinants of heart rate variability. Int Arch Occup Environ Health 2000;73:255-62.

7 Amelsvoort LGPM van, Schouten EG, Maan AC, et al. 24-Hour heart rate variability in shift workers: impact of shift schedule. F Occup Health 2001;43:32-8.

8 Härenstam A, Theorell T, Orth-Gomer K, et al. Shift work, decision latitude and ventricular ectopic activity: a study of 24-hour electrocardiograms in Swedish prison personnel. Work Stress 1987;1:341-50

9 Theorell T, Åkerstedt T. Day and night work: changes in cholesterol, uric acid, glucose and potassium in serum and in circadian patterns of urinary catecholamine excretion. Acta Med Scand 1976;200:47-53.

10 Sgoifo A, de Boer SF, Westenbroek C, et al. Incidence of arrhythmias and heart rate variability in wild-type rats exposed to social stress. Am F Physiol 1997;273:H1754760 .

11 Lown B, DeSilva RA, Lenson R. Roles of psychologic stress and autonomic nervous system changes in provocation of ventricular premature complexes. Am f Cardiol 1978;41: 979-85.

\title{
7th European Forum on Quality Improvement in Health Care
}

\author{
21-23 March 2002 \\ Edinburgh, Scotland
}

We are delighted to announce this forthcoming conference in Edinburgh. Authors are invited to submit papers (call for papers closes on Friday 5 October 2001) and delegate enquiries are welcome.

The themes of the Forum are:

- Leadership, culture change, and change management

- Achieving radical improvement by redesigning care

- Health policy for lasting improvement in health care systems

- Patient safety

- Measurement for improvement, learning, and accountability

- Partnership with patients

- Professional quality: the foundation for improvement

- Continuous improvement in education and training

- People and improvement.

Presented to you by the BMJ Publishing Group (London, UK) and Institute for Healthcare Improvement (Boston, USA). For more information contact: quality@bma.org.uk or look at the website www.quality.bmjpg.com. Tel: +44 (0)20 7383 6409; fax: +44 (0)20 73736869 . 